\title{
Minimizing DC System Loss in Multi-Terminal HVDC Systems through Adaptive Droop control
}

\author{
Javad Khazaei, Zhixin Miao, Lakshan Piyasinghe, and Lingling Fan* \\ Department of Electrical Engineering, University of South Florida, Tampa FL USA 33620. \\ Phone: 1(813)974-2031, Fax: 1(813)974-5250,Email: linglingfan@usf.edu.
}

\begin{abstract}
In this paper, droop control of inverters in a multi-terminal HVDC (MTDC) system is designed to achieve minimum DC system loss while preventing converter overvoltage. Circuit analysis along with optimization analysis is first conducted to seek the optimal droop control gains. Contingent operating conditions are then considered. The droop control gains will adapt to achieve minimum loss as well as constant inverter side dc voltage. A 6-terminal MTDC system with detailed control loops as well as PWM-based switching is built in a real-time digital simulator, RT-Lab. Time-domain simulation results validate the circuit analysis results and demonstrate the feasibility of the proposed adaptive droop control.
\end{abstract}

Keywords: Multi Terminal HVDC (MTDC), Droop Control, Minimization of Losses.

\section{Introduction}

Multi-terminal HVDC (MTDC) systems have been proposed to deliver offshore wind power $[1,2,3,4,5]$. The basic configuration of the MTDC system is illustrated in Fig. 1. The MTDC system is composed of two parts: wind farm side and grid side. At the wind farm side, a rectifier will be used to convert ac electricity to dc electricity. A dc cable is used to join the terminals to the sending end (point $S$ ). Electricity will be transferred through a dc cable to the receiving end point (point $R$ ), and finally reaching inverter terminals. At the inverters terminal, electricity will be converted from dc back to ac.

The inverter controls have to be coordinated for power sharing. There are two well-known methods for inverter control coordination: master-slave control [6] and droop control $[7,5]$. In the master-slave control paradigm, one inverter (master) will be controlled in constant dc voltage mode and served as a dc voltage source. The rest of the inverters (slaves) can be controlled in power mode. Therefore, all other inverters except the master can have scheduled power outputs while the master serves as a slack bus to absorb the rest of the power. It is required that the master inverter has a high capacity so it can accommodate power. 
The master-slave control has reliability issues. When the master converter is lost, the entire system will shut down.

On the other hand, in droop control, inverters share power based on their droop control characteristics. If one inverter is lost, the entire system can still function $[7,5]$. Therefore, variety of droop schemes have been proposed for MTDC. In [8, 9], active power-dc voltage droop control is proposed. In the vector control implementation for a converter, the $d$-axis current reference is generated by a proportional-integral (PI) controller from dc voltage control. The reference of the voltage square is modified by active power droop $\left(V_{\mathrm{dc}}^{2 \mathrm{ref}}=V_{\mathrm{dc}}^{2 *}+\beta\left(P^{*}-P\right)[8]\right)$. In [10], dc current-dc voltage droop control is proposed $\left(V_{\mathrm{dc}}^{\mathrm{ref}}=V_{\mathrm{dc} 0}+K I_{\mathrm{dc}}\right)$.

Appropriate design of droop control can not only facilitate power sharing among inverters, but also achieve other functions, e.g., robust dynamic performance, post-contingent power sharing, and loss minimization. For example, in [7, 11], robust control method is adopted to design droop control for MTDC. Instead of using fixed values for droop control gains, [8] proposes to use different droop control gains at post-contingency operating conditions so that each inverter has an adequate sharing of "headroom" (difference between the rated capacity and present loading). In [10], a methodology is proposed to minimize the copper losses in dc cables by keeping all the dc voltages at the grid side constant and selecting the droop gains proportional to the corresponding cable resistances.

Minimizing dc cable loss has been also addressed in [12]. The proposed method is to find out each inverter's de side setting point through an optimization algorithm. And the inverters are controlled in constant dc voltage mode. Droop control is not in the picture. The proposed "optimum voltage control" can minimize the loss, however, it cannot fulfill the task of power sharing.

This paper aims to design droop control for MTDC systems to have the function of power sharing, at the same time, to achieve minimum dc system loss. Research on loss minimization of MTDC systems has been seen in the literature. For example, optimal power flow algorithms to include MTDC are discussed in $[13,14,11]$. In [13], an optimal power flow problem is formulated to minimize the loss in the AC system. DC system is posed as constraints. In [14], different optimal power flow approaches for MTDC systems are summarized. Effect of droop control in MTDC systems on the entire ac/dc system power flow as well as optimal power flow has also been discussed in $[14,13]$. The proposed research of this paper is limited to dc systems. Instead of viewing this research as a power flow computation problem, we view this problem as a dynamic control problem. Therefore, dc system voltage constraints as well as contingent scenarios will be considered.

The droop control adopted in this paper is dc current-dc voltage droop control similar as those presented 
in [10]. Advantage of this type of droop compared to power-dc voltage droop used in $[8,9]$ is that the droop control can be considered as a Thevenin equivalent voltage source behind a resistor ( where the resistance equals the droop gain). On the other hand, impact on the dc system power/currents due to the power-dc voltage type droop control has to be investigated through more complicated analysis, as demonstrated in [9].

Compared to the research on droop control-based system loss minimization in the literature, e.g., [10], the contributions of this paper are listed as follows.

1. A comprehensive circuit analysis including the rectifier terminals, the inverter system terminals, and the effect of droop gains has been carried out. Our research not only indicates that the inverter droop gains should be proportional to the corresponding cable resistance similar as the conclusion drawn in [10], but also proposes a procedure to compute the droop gains. We have also presented an important finding: in order for the system to achieve minimum loss, one of the rectifier terminals should have the maximum allowed voltage level.

2. Droop gain computation for abnormal scenarios is proposed to keep the inverter terminal dc voltages constant.

3. While the research in the literature focuses on circuit analysis [10], this paper not only gives circuit analysis results but also validates analytical results through detail model based simulation. In our research, converter controls and PWM dynamics have been included in the simulation model. Therefore, the verification is more realistic.

The rest of the paper is organized as the follows. Section 2 describes the operation and control of the MTDC systems. Circuit analysis and optimization analysis are carried out in Section 3 to determine the best choice of droop gains for inverters. A subsection is dedicated to analyze an abnormal scenario where one converter at the grid side is lost. Simulation results are covered in Section 4 and conclusion remarks are presented in Section 5 .

\section{Operation and Control of a MTDC System}

The general structure of the MTDC system with $m$ terminals at the rectifier side and $n$ terminals at the inverter side is illustrated in Fig. 1. Each ac grid is modeled as a voltage source connected to an MTDC converter through a series-connected inductor. For the dc system, each terminal is connected to a dc cable and these cables will be connected to a common coupling point. A dc cable will connect the rectifier side and the inverter side together. 
The rectifiers are controlled in the mode to transmit power that is generated. The inverters are in charge of controlling their dc voltage levels as well as reactive power to the ac systems. The voltage droop control is implemented to adjust the dc voltage reference. Such a control diagram is shown in Fig. 1. Both inverter and rectifier controls are generating pulses through an inner current control loop. The PWM unit for each converter aligns the output converter voltage with the ac side voltage using a Phase-Locked-Loop (PLL).

\subsection{Rectifier Control}

For the rectifier side of the MTDC, the control mode is set to $P-Q$ control in order to control the active and reactive powers of the generation unit independently. The control system of the rectifier of the MTDC is shown in Fig. 3 .

In Fig. $3, \omega_{s}$ is the angular frequency of the ac system, $V_{A C w 1}$ represents the vector of the point of common coupling (PCC) instantaneous $a b c$ phase voltages. In addition, $E_{d}$ represents the d-axis voltage from $V_{A C w 1}$ after $a b c / d q$ conversion $\left(E_{q}=0\right.$ due to the alignment of the reference frame with the PCC voltage). Furthermore, $V_{a}, V_{b}$ and $V_{c}$ represent the converter output voltage, $i_{d}$ and $i_{q}$ represent the $d$ - and $q$-axis currents flowing to the VSC respectively. This control system consists of two loops: inner current control loop and outer power control loop. The parameters of the controllers are given in Appendix.

\subsection{Inverter Control}

At the inverter station, the control mode is set to control the dc voltage and reactive power to the ac grid. The droop control is used to share the active power among the inverters. The droop control modifies the reference dc voltage at each grid-side converter. A PI controller is used to regulate the dc voltage to follow the reference( $\left.V_{\mathrm{DC}}^{\text {ref }}=V_{\mathrm{DC} 0}+K I_{\mathrm{DC}}\right)$. The control system is shown in Fig. 4 .

\section{Circuit Analysis and Optimal Setting of Droop Gains}

The objective of this research is to set the droop gains to achieve minimum dc system loss. Circuit analysis and optimization will be carried out in this section. We start by deriving the equivalent circuit of the MTDC system, which is illustrated in Fig. 2(a).

The dc cables are modeled as resistors. It is noted that, $E_{w_{i}}$ and $E_{g_{i}}$ represent the dc voltages of the wind farm terminals and voltages of the grid terminals, respectively. The Thevenin circuit including the droop gain effect is also illustrated in Fig. 2(a), where $V_{\mathrm{DC} 0}$ is the reference dc voltage used in inverter controls. 
The system can be further simplified as Fig. 2(b), where the Thevenin equivalent voltages and resistances are expressed as follows.

$$
\begin{aligned}
& E_{g_{t h}}=\frac{\sum_{j=1}^{n} \frac{E_{g_{j}}}{R_{g_{j}}}}{\sum_{j=1}^{n} \frac{1}{R_{g_{j}}}} \\
& R_{g_{t h}}=\frac{1}{\sum_{j=1}^{n} \frac{1}{R_{g_{j}}}} \\
& E_{w_{t h}}=\frac{\sum_{i=1}^{m} \frac{E_{w_{i}}}{R_{w_{i}}}}{\sum_{i=1}^{m} \frac{1}{R_{w_{i}}}} \\
& R_{w_{t h}}=\frac{1}{\sum_{i=1}^{m} \frac{1}{R_{w_{i}}}}
\end{aligned}
$$

The dc system loss is composed of three components: the loss due to $R_{w_{i}}$, the loss due to $R_{S R}$, and the loss due to $R_{g i}$. Since the cable between points $S$ and $R$ is the major transmission link, loss on this cable will be the major component of the total loss. Therefore, in order to have minimum dc system loss, it is desirable to have minimum $I_{t}$.

\subsection{Rectifier Side Analysis}

Given a fixed power level $P_{w_{i}}$ from a converter terminal, the dc current $I_{w_{i}}$ will be minimum when the dc voltage level $E_{w_{i}}$ reaches its maximum. Since the voltage at the joint point $S\left(E_{s}=E_{w_{i}}-R_{w_{i}} \frac{P_{w_{i}}}{E_{w_{i}}}\right)$ depends on every terminal voltage $E_{w_{i}}$, if more than one terminal voltages are scheduled, there will be conflicts for $E_{s}$. Therefore, only one terminal will have the maximum voltage level.

An iterative method is carried out to search for the rectifier terminal that should be on the maximum level at each operating point. After finding that terminal, a step by step procedure is used to find the desired $I_{t}$. The procedure is listed:

- Active power at each rectifier terminal is given $\left(P_{w_{i}}\right.$ for $\left.i=1,2, \ldots, m\right)$.

- After finding one rectifier that should work on its maximum voltage ( $E_{w_{k}}$ is assumed), the converter's DC current is derived by: $I_{w_{k}}=\frac{P_{w_{k}}}{E_{w_{k}}}$. 
- The sending end voltage is then derived by:

$$
E_{s}=E_{w_{k}}-R_{w_{k}} I_{w_{k}}
$$

- $I_{t}$ is computed by the following equations.

Since $E_{w_{i}}=E_{S}+R_{w_{i}} \frac{P_{w_{i}}}{E_{w_{i}}}$, we can find the expression of $I_{w_{i}}$ in terms of $P_{w_{i}}$ and $E_{S}$ as

$$
I_{w_{i}}=\frac{-E_{S}+\sqrt{E_{S}^{2}+4 R_{w_{i}} P_{w_{i}}}}{2 R_{w_{i}}}
$$

Therefore,

$$
I_{t}=\sum_{i=1}^{m} I_{w_{i}}=\sum_{i=1}^{m}\left(\frac{-E_{S}+\sqrt{E_{S}^{2}+4 R_{w_{i}} P_{w_{i}}}}{2 R_{w_{i}}}\right)
$$

- The receiving end voltage $E_{R}$ as well as the Thevenin equivalent terminal voltage $E_{g_{t h}}$ can also be found.

The rectifier side analysis gives the desired total current $I_{t}$, the desired rectifier side terminal voltages $E_{w_{i}}$, the desired $E_{S}$, the desired $E_{R}$ and the desired $E_{g_{t h}}$.

Note that the rectifier control only determines the scheduled power level $P_{w_{i}}$. Therefore, the rectifier control alone cannot determine the steady-state dc currents and voltage levels. It is left to the inverter control, specifically droop gains, to determine the steady-state dc currents and voltage levels.

\subsection{Inverter Side Analysis}

Given the desired $I_{t}$, the next task is to allocate current to each inverter to have minimum loss on the cables connecting the inverters. The loss related to the inverter-side cables can be written as

$$
P_{\text {loss }}=\sum_{j=1}^{n} R_{g_{j}} I_{g_{j}}^{2}
$$

Further modification will lead to:

$$
P_{\text {loss }_{g}}=\sum_{j=1}^{n-1} R_{g_{j}} I_{g_{j}}^{2}+R_{g_{n}}\left(I_{t}-\sum_{k=1}^{n-1} I_{g_{k}}\right)^{2} .
$$


In order to define the optimal solution of current allocation $I_{g_{j}}^{*}$, the partial derivative of losses versus any $I_{g_{j}}$ evaluated at $I_{g_{j}}^{*}$ should be zero.

$$
\begin{gathered}
\left.\frac{\partial P_{\text {loss }_{g}}}{\partial I_{g_{j}}}\right|_{I_{g_{j}}^{*}}=2 R_{g_{j}} I_{g_{j}}^{*}-2 R_{g_{n}}\left(I_{t}-\sum_{k=1}^{n-1} I_{g_{k}}^{*}\right)=0 \\
\Rightarrow R_{g_{j}} I_{g_{j}}^{*}=R_{g_{n}}\left(I_{t}-\sum_{k=1}^{n-1} I_{g_{k}}^{*}\right) \text { for all } j=1, \ldots, n-1
\end{gathered}
$$

Combining $I_{t}=\sum_{i=1}^{m} I_{w_{i}}=\sum_{j=1}^{n} I_{g_{j}}$ and the $n-1$ equations of (10) results in the following finding:

$$
\begin{aligned}
& R_{g_{j}} I_{g_{j}}^{*}=R_{g_{n}} I_{g_{n}}^{*} \\
& E_{g_{j}}^{*}=E_{g_{n}}^{*}=E_{g_{t h}} \quad \text { for all } j=1, \ldots, n .
\end{aligned}
$$

It proves that to minimize the losses at the inverter side, the terminal voltage of the converters should be equal. This finding has also been documented in [10].

The finding indicates that the active power sharing among inverters is proportional to the current sharing:

$$
\frac{P_{g j}}{P_{g k}}=\frac{E_{g j} \cdot I_{g j}}{E_{g k} \cdot I_{g k}}=\frac{I_{g j}}{I_{g k}}
$$

In addition, based on the voltage/current relationship for the circuit in Fig. 2(a) $\left(E_{g_{j}}=V_{\mathrm{DC} 0}+K_{j} I_{g_{j}}\right.$ and $E_{g_{j}}=E_{R}-I_{g_{j}} R_{g_{j}}$, the currents are inversely proportional to the droop gains and the cable resistances.

$$
\frac{P_{g j}}{P_{g k}}=\frac{I_{g j}}{I_{g k}}=\frac{R_{g k}}{R_{g j}}=\frac{K_{k}}{K_{j}}
$$

The important finding from the above analysis is that the droop gains should be selected proportional to the cable resistances $\left(K_{j} \propto R_{j}\right)$.

The calculation of the droop gain $K_{j}$ can be obtained by further analysis. Since the droop gains are proportional to the cable resistances, the current sharing among inverters will be inversely proportional to the cable resistance at each branch. Therefore,

$$
I_{g_{j}}=\frac{\frac{1}{R_{g_{j}}}}{\sum_{k=1}^{n} \frac{1}{R_{g_{k}}}} I_{t}
$$


The droop gains can be computed based on $E_{g_{j}}=E_{g_{t h}}$ and $I_{g_{j}}$.

$$
K_{j}=\frac{E_{g_{j}}-V_{\mathrm{DC} 0}}{I_{g_{j}}}
$$

The proposed algorithm for droop gain calculation works for any operating point. It can ensure the minimum total current $\left(I_{t}\right)$ from the rectifier side analysis.

Remarks:

1. When the power level $P_{w_{i}}$ changes, the droop gains should be recalculated. This is due to the fact that $I_{t}$ depends on $P_{w_{i}}$ and the droop gain depends on $I_{t}$.

2. When the system topology changes, e.g., a grid-side converter is lost, the droop gains should be recalculated.

In the following subsection, we will compute the droop gains when one of the grid-side converters is lost.

\subsection{Analysis of an Abnormal System}

To conduct the analysis, we assume that one inverter is lost. A line trip detection algorithm is defined in the proposed method. When a line trip happens in a terminal, a fault signal should be sent to the algorithm to recalculate the droop gains based on the new condition. Fault signal is a binary variable: 1 if fault happens and 0 when there is no fault. To initiate the fault analysis, the DC current at each terminal is continuously measured and sent to a low pass filter with a cut off frequency of $5 \mathrm{~Hz}$. The output of the filter is then compared with a threshold $(10 \mathrm{~A})$. If the filter output current is less than $10 \mathrm{~A}$, line trip is detected. The fault signal along with the fault bus index will be sent to droop calculation procedure. If a fault happens in a specific terminal, that terminal will be removed from droop calculation. Droop gains for the other terminals will be updated based on total DC current calculated at rectifier stations.For example, if the line trip happens at bus 3 , the power levels are intact. The desired total current $I_{t}$ and the desired grid terminal voltage $E_{g_{t h}}=E_{g_{j}}$ will be the same. Therefore the current allocation to each converter will change at the grid side.

$$
\begin{aligned}
& I_{g_{1}}=\frac{\frac{1}{R_{g_{1}}}}{\frac{1}{R_{g_{1}}}+\frac{1}{R_{g_{2}}}} I_{t} \\
& I_{g_{2}}=\frac{\frac{1}{R_{g_{2}}}}{\frac{1}{R_{g_{1}}}+\frac{1}{R_{g_{2}}}} I_{t}
\end{aligned}
$$


The droop gains will be found from the following equations.

$$
\begin{aligned}
& K_{1}=\frac{E_{g_{1}}-V_{\mathrm{DC} 0}}{I_{g_{1}}} \\
& K_{2}=\frac{E_{g_{2}}-V_{\mathrm{DC} 0}}{I_{g_{2}}}
\end{aligned}
$$

\section{Case Studies}

The study system is built in RT-LAB, a real-time digital simulator manufactured by OPAL-RT, which can simulate the power system model with detailed power electrotonic switches in real-time [15]. Therefore, it can provide precise simulation results and take the switching details of IGBTs into account.

The test system is a 6-terminal MTDC system composed of three rectifier side terminals and three inverter side terminals as shown in Fig. 1. The base voltage is set to $250 \mathrm{kV}$ and the base power is set to 100 MW. Fixed generated power is considered at each rectifier side which is set to 100, 90, and $110 \mathrm{MW}$, respectively.

The maximum voltages for converters are considered as $260 \mathrm{kV}$ and maximum current settings of the converters are set to $1 \mathrm{kA}$. The optimal droop gains are computed and listed in Table 1 . Also listed are the terminal voltages and currents. The dc voltage setting in the inverter control $V_{\mathrm{DC} 0}$ is $240 \mathrm{kV}$.

It is observed that at the inverter side, all the inverters are working at the same voltage of $255 \mathrm{kV}$. The total current through the major dc cable $S R\left(I_{t}\right)$ is $1142 \mathrm{~A}$. Furthermore, at the rectifier side, as the maximum voltage for all the converters are the same, all of them are working at their maximum. This will ensure the maximum power transmission through MTDC system as well as minimum loss condition.

\subsection{One of the inverter side terminals is tripped}

In this case study, we study the effect of adaptive droop gains when one inverter is tripped. Two cases are considered here:

- Case 1: the droop gains will not be updated after the trip.

- Case 2: the droop gains will be updated following the algorithm proposed in Section 3.3.

In Case 1, the droop gains are obtained from the proposed algorithm as $K_{1}=41.89, K_{2}=35$, and $K_{3}=48.82$ for the nominal operating condition. Further, the gains are fixed. The steady-state voltages and currents after an inverter is tripped are listed in Table 2. It can be seen from circuit calculation that the dc voltages all rise up. Dynamic simulation results will be presented following the circuit analysis results. 
Results of voltages at rectifier and inverter side have been shown in Fig. 5 . After the fault at 5 sec, the voltages at both sides will rise. The maximum voltage for the rectifier side before the fault is set to $260 \mathrm{kV}$. After the fault, the voltage levels reached $265 \mathrm{kV}$. This $5 \mathrm{kV}$ increase in the voltage will cause damage to converters.

Results of the rectifier side and inverter side currents for Case 1 have been illustrated in Fig. 6. As observed, the current for the third terminal eventually reaches zero. Oscillations around zero are observed during the transients. This is because the current is measured before the dc-link of the converters. The oscillations are absorbed by the dc-link capacitors.

In Case 2, the proposed algorithm will update the droop gains at each different operating point in order to guarantee the minimum loss in the system. In the normal operation, the proposed algorithm will result in optimized steady state droop gains as: $K_{1}=41.89, K_{2}=35$, and $K_{3}=48.82$. After 5 seconds, the contingency is considered as a three-phase line trip in the third inverter side terminal. The droop gains are recomputed and listed in Table 3 .

Change of droop gains has been illustrated in Fig. 7. After the fault, the third terminal is out and its droop gain for this terminal is shown as zero. There is a small delay between the line trip (5 sec) and the time that droop change is applied refer to Fig. 7. This transient is the time needed for the fault detection algorithm to detect the fault.

Simulation results of the terminal voltages at the inverter side and rectifier side are illustrated in Fig. 8. Simulation results for the currents are shown in Fig. 9. It can be observed that after the fault at 5 sec, the rectifier side and inverter side voltages are kept the same as they were before the fault. As the rectifier side converters are working on their maximum voltage limit, the rectifier side is guaranteed to work on minimum loss. Furthermore, at the inverter side, all the terminal voltages are equal, which ensures the minimum losses in the inverter side. The other important result is that the dc voltages did not rise up after the fault. It shows that the proposed algorithm not only ensures the minimum losses in the MTDC system, but also ensures constant dc voltage level.

\subsection{Change of active power generated from wind farms}

In this case study, we examine the effect of active power change on droop gain calculation and resulting system performance. Generation power of one rectifier side terminal is increased from $90 \mathrm{MW}$ to $120 \mathrm{MW}$ at 5 seconds. The other two terminals' power are kept the same (100 and $110 \mathrm{MW})$. Two cases are compared.

- Case 3: the droop gains will not be updated after the power change. 
- Case 4: the droop gains will be updated following the algorithm proposed in Section 3.

The droop gains, dc voltages and currents at steady-state for Case 3 is listed in Table 4 . It can be seen that the wind side terminals will have over voltages.

Fig. 10 shows the terminal voltages of rectifier side and inverter side when fixed droop gains are used. The optimal droop gains are used in this case same as previous cases $\left(K_{1}=41.89, K_{2}=35\right.$, and $\left.K_{3}=48.82\right)$. After the change in power, the droop gains will be kept the same. As it can be observed, both terminals' voltages will rise up after the operating point change.

In Case 4, the droop gains are recomputed for a changed power level. The results are listed in Table 5. It can be observed that the steady-state dc voltage levels are kept the same as that of the base case. Simulation studies for the optimized case where droop gains are updated are also carried out. The simulation results are illustrated in Fig. 11. It is observed that with power change at one terminal, the proposed adaptive droop gain strategy can keep the voltages of inverter side and rectifier side constant.

\subsection{Fixed droop gains}

In this case study, the droop gains are selected in a way that when all three grid side converters are in operation. Moreover, the converter voltages are well below the maximum converter voltage limit. Without considering the converter limits, if the droop gains are selected randomly, when a fault happens, converter voltages will rise above the maximum limit and makes the converter unstable. Instead, here the droop gains are considered so that even if a fault happens (in the worst case), voltage rise due to the fault will not be greater than the converter limit and the system is stable. System calculations for the non-optimized case 5 are included in Table 6.

Knowing the currents in DC grid will lead to find all the losses. For example, rectifier side loss is

calculated by $\sum_{i=1}^{m} R_{w i} I_{w i}^{2}$. The inverter side loss is $\sum_{i=1}^{n} R_{g i} I_{g i}^{2}$. The loss at the main DC cable $S R$ is: $R_{S R} I_{t}^{2}$. Total loss is calculated by adding these three values. For the non-optimized case with conservative droop selected, (case 5), total current is $\left(I_{t}=1167 A\right.$ ), and total losses are calculated to be $5.3 \mathrm{MW}$ (Loss on the rectifier side: $0.77 \mathrm{MW}$, loss on the inverter side: $0.75 \mathrm{MW}$, and loss on the tie line:3.78 $M W$ ).

If one of the inverter side converters is disconnected due to a three phase-fault at AC grid, the voltage of other converters will rise. The droop gains are kept the same. Table 2 gives the steady-state voltage and current values after the fault. Since conservative droop gains have been selected in order to keep the voltages well below the limits, voltages of grid side converters after the fault would not surpass the maximum voltage limit (260 kV in this case). The total loss is $5.5 \mathrm{MW}$ (loss of the rectifier side: $0.77 \mathrm{MW}$, loss of the grid 
side: $1.03 \mathrm{MW}$, and tie line loss: $3.70 \mathrm{MW}$ ).

For the optimized case before the line trip, total current is $\left(I_{t}=1142 \mathrm{~A}\right)$, and total losses are calculated to be $5.08 \mathrm{MW}$ (loss on the rectifier side: $0.75 \mathrm{MW}$, loss on the grid side: $0.71 \mathrm{MW}$, and loss on the tie line: $3.62 \mathrm{MW}$ ). The optimized droop gains are in proportional with the resistances of the line at each moment. Total losses have been decreased from 5.3 $\mathrm{MW}$ to 5.08 $\mathrm{MW}$ compared to non-optimized case.

For the contingent case (Case 2), the total loss is $5.31 \mathrm{MW}$ (loss of the rectifier side: $0.74 \mathrm{MW}$, loss of the inverter side:e $0.98 \mathrm{MW}$, and tie line loss: $3.59 \mathrm{MW}$ ). This is also less than the loss of the non-optimized case. It is worth mentioning that all the voltages are kept within the limit and proposed optimal power flow algorithm has guaranteed the minimum loss condition even when the fault happened.

\section{Conclusion}

This paper proposes a droop control-based dc system loss minimization strategy. Through setting droop gains for grid-side converters according to the proposed algorithm for every operating condition, the MTDC system can achieve minimum dc loss and constant dc voltage level for changing operating conditions. The change in operating conditions can be the increase in power transfer or loss of a grid-side converter. The proposed droop gain computation algorithm has two notable features: (i) droop gains are proportional to the cable resistances; (ii) one of the wind farm terminals will work at maximum voltage level. Real-time digital simulation-based tests were carried out to demonstrate the proposed adaptive droop control in keeping dc voltage level and minimizing dc loss with changing operating conditions.

\section{References}

[1] J. Liang et al., "Control of multi-terminal vsc-hvdc transmission for offshore wind power," in 2009 13th European Conference on Power Electronics and Applications, 2009, pp. 1-10.

[2] D. Van Hertem and M. Ghandhari, "Multi-terminal vsc hvdc for the european supergrid: Obstacles," Renewable and sustainable energy reviews, vol. 14, no. 9, pp. 3156-3163, 2010.

[3] J. Liang, T. Jing, O. Gomis-Bellmunt, J. Ekanayake, and N. Jenkins, "Operation and control of multiterminal HVDC transmission for offshore wind farms," Power Delivery, IEEE Transactions on, vol. 26, no. 4, pp. 2596-2604, 2011.

[4] L. Xu and L. Yao, "Dc voltage control and power dispatch of a multi-terminal hvdc system for integrating large offshore wind farms," IET renewable power generation, vol. 5, no. 3, pp. 223-233, 2011.

[5] E. Prieto-Araujo, F. D. Bianchi, A. Junyent-Ferre, and O. Gomis-Bellmunt, "Methodology for droop control dynamic analysis of multiterminal vsc-hvdc grids for offshore wind farms," Power Delivery, IEEE Transactions on, vol. 26, no. 4, pp. 2476-2485, 2011.

[6] J. Beerten, S. Cole, and R. Belmans, "Generalized steady-state vsc mtdc model for sequential ac/dc power flow algorithms," Power Systems, IEEE Transactions on, vol. 27, no. 2, pp. 821-829, 2012. 
[7] F. D. Bianchi and O. Gomis-Bellmunt, "Droop control design for multi-terminal vsc-hvdc grids based on lmi optimization," in Decision and Control and European Control Conference (CDC-ECC), 2011 50th IEEE Conference on. IEEE, 2011, pp. $4823-4828$.

[8] N. R. Chaudhuri and B. Chaudhuri, "Adaptive droop control for effective power sharing in multi-terminal dc (mtdc) grids," Power Systems, IEEE Transactions on, vol. 28, no. 1, pp. 21-29, 2013.

[9] T. M. Haileselassie and K. Uhlen, "Impact of dc line voltage drops on power flow of mtdc using droop control," Power Systems, IEEE Transactions on, vol. 27, no. 3, pp. 1441-1449, 2012.

[10] A. S. Abdel-Khalik, A. M. Massoud, A. A. Elserougi, and S. Ahmed, "Optimum power transmission-based droop control design for multi-terminal hvdc of offshore wind farms," Power Systems, IEEE Transactions on, vol. 28, no. 3, pp. 3401$3409,2013$.

[11] A. Egea-Alvarez, F. Bianchi, A. Junyent-Ferré, G. Gross, and O. Gomis-Bellmunt, "Voltage control of multiterminal VSCHVDC transmission systems for offshore wind power plants: Design and implementation in a scaled platform," Industrial Electronics, IEEE Transactions on, vol. 60, no. 6, pp. 2381-2391, 2013.

[12] M. Aragüés-Peñalba, A. Egea-Alvarez, O. Gomis-Bellmunt, and A. Sumper, "Optimum voltage control for loss minimization in hvdc multi-terminal transmission systems for large offshore wind farms," Electric Power Systems Research, vol. 89, pp. 54-63, 2012.

[13] J. Cao, W. Du, H. Wang, and S. Bu, "Minimization of transmission loss in meshed ac/dc grids with vsc-mtdc networks," Power Systems, IEEE Transactions on, vol. 28, no. 3, pp. 3047-3055, Aug 2013.

[14] W. Wang and M. Barnes, "Power flow algorithms for multi-terminal VSC-HVDC with droop control," Power Systems, IEEE Transactions on, vol. 29, no. 4, pp. 1721-1730, July 2014.

[15] S. Abourida, C. Dufour, J. Bélanger, G. Murere, N. Léchevin, and B. Yu, "Real-time pc-based simulator of electric systems and drives," in Applied Power Electronics Conference and Exposition, 2002. APEC 2002. Seventeenth Annual IEEE, vol. 1. IEEE, 2002, pp. 433-438.

\section{Table of Tables}

Table 1: Droop gains at the base case

\begin{tabular}{|l|l|l|l|l|l|l|}
\hline \multicolumn{1}{c}{ Inverter Side } & \multicolumn{4}{c|}{ Rectifier Side } \\
\hline Converter & Droop & $R_{g}(\Omega)$ & $E_{g}(\mathrm{kV})$ & $I_{g}(\mathrm{~A})$ & $E_{w}(\mathrm{kV})$ & $I_{w}(\mathrm{~A})$ \\
\hline 1 & 41.89 & 1.69 & 255 & 375 & 260 & 380 \\
\hline 2 & 35 & 1.39 & 255 & 447 & 260 & 342 \\
\hline 3 & 48.82 & 1.95 & 255 & 320 & 260 & 420 \\
\hline
\end{tabular}

Table 2: Droop gains, voltages and currents for Case 1 non-adaptive droops after line trip

\begin{tabular}{|l|l|l|l|l|l|l|}
\hline \multicolumn{4}{c}{ Inverter Side } & \multicolumn{3}{c}{ Rectifier Side } \\
\hline Converter & Droop & $R_{g}(\Omega)$ & $E_{g}(\mathrm{kV})$ & $I_{g}(\mathrm{~A})$ & $E_{w}(\mathrm{kV})$ & $I_{w}(\mathrm{~A})$ \\
\hline 1 & 41.89 & 1.69 & 261 & 510 & 265 & 375 \\
\hline 2 & 35 & 1.39 & 261 & 614 & 265 & 337 \\
\hline 3 & - & 1.95 & 261 & 0 & 265 & 412 \\
\hline
\end{tabular}

\section{Table of Figures}

1. A 6-terminal MTDC. AC voltage level: $100 \mathrm{kV}$; DC voltage level: $250 \mathrm{kV}$. $C=350 \mu \mathrm{F}$, DC cables have the same resistance: $2.78 \times 10^{-2} \Omega / \mathrm{km}$, DC cable lengths: Rectifier1: $10 \mathrm{~km}$, Rectifier2: $20 \mathrm{~km}$, 
Table 3: Droop gains, voltages and currents for Case 2 adaptive droop gains after the line trip

\begin{tabular}{|l|l|l|l|l|l|l|}
\hline \multicolumn{9}{c}{ Inverter Side } & \multicolumn{3}{c|}{ Rectifier Side } \\
\hline 1 & 29.7 & 1.69 & 255 & 517 & 260 & 380 \\
\hline 2 & 24.7 & 1.39 & 255 & 620 & 260 & 340 \\
\hline 3 & - & 1.95 & 255 & 0 & 260 & 417 \\
\hline
\end{tabular}

Table 4: Droop gains, voltages and currents for Case 3 non-adaptive droop for active power change

\begin{tabular}{|l|l|l|l|l|l|l|}
\hline \multicolumn{4}{c}{ Inverter Side } & \multicolumn{3}{c|}{ Rectifier Side } \\
\hline Converter & Droop & $R_{g}(\Omega)$ & $E_{g}(\mathrm{kV})$ & $I_{g}(\mathrm{~A})$ & $E_{w}(\mathrm{kV})$ & $I_{w}(\mathrm{~A})$ \\
\hline 1 & 41.89 & 1.69 & 257 & 400 & 262 & 380 \\
\hline 2 & 35 & 1.39 & 257 & 483 & 262 & 428 \\
\hline 3 & 48.82 & 1.95 & 257 & 345 & 262 & 420 \\
\hline
\end{tabular}

Table 5: Droop gains, voltages and currents at steady-state for Case 4 adaptive droop gains for active power change

\begin{tabular}{|l|l|l|l|l|l|l|}
\hline \multicolumn{4}{c}{ Inverter Side } & \multicolumn{3}{c|}{ Rectifier Side } \\
\hline Converter & Droop & $R_{g}(\Omega)$ & $E_{g}(\mathrm{kV})$ & $I_{g}(\mathrm{~A})$ & $E_{w}(\mathrm{kV})$ & $I_{w}(\mathrm{~A})$ \\
\hline 1 & 37.1 & 1.69 & 255 & 413 & 260 & 385 \\
\hline 2 & 30.9 & 1.39 & 255 & 493 & 260 & 457 \\
\hline 3 & 43.3 & 1.95 & 255 & 356 & 260 & 420 \\
\hline
\end{tabular}

Table 6: Droop gains, voltages and currents at steady-state for case 5 non-adaptive droop gains before the fault Inverter Side Rectifier Side

\begin{tabular}{|l|l|l|l|l|l|l|}
\hline Converter & Droop & $R_{g}(\Omega)$ & $E_{g}(\mathrm{kV})$ & $I_{g}(\mathrm{~A})$ & $E_{w}(\mathrm{kV})$ & $I_{w}(\mathrm{~A})$ \\
\hline 1 & 30 & 1.69 & 250 & 337 & 254 & 390 \\
\hline 2 & 20 & 1.39 & 250 & 497 & 254 & 349 \\
\hline 3 & 30 & 1.95 & 250 & 333 & 254 & 428 \\
\hline
\end{tabular}

Table 7: Droop gains, voltages and currents at steady-state for case 6 non-adaptive droop gains after the fault

\begin{tabular}{|l|l|l|l|l|l|l|}
\hline \multicolumn{4}{c}{ Inverter Side } & \multicolumn{3}{c}{ Rectifier Side } \\
\hline Converter & Droop & $R_{g}(\Omega)$ & $E_{g}(\mathrm{kV})$ & $I_{g}(\mathrm{~A})$ & $E_{w}(\mathrm{kV})$ & $I_{w}(\mathrm{~A})$ \\
\hline 1 & 30 & 1.69 & 254 & 465 & 258 & 382 \\
\hline 2 & 20 & 1.39 & 254 & 690 & 258 & 348 \\
\hline 3 & - & 1.95 & 254 & 0 & 258 & 425 \\
\hline
\end{tabular}

Table 8: Parameters of the wind farm side Rectifiers

Table 8: Parameters of the wind farm side Rectifie
\begin{tabular}{|c|c|}
\hline$K_{p}$ current control loop & 50 \\
\hline$K_{i}$ current control loop & 100 \\
\hline$L$ & $0.1 \mathrm{H}$ \\
\hline$Q_{\text {ref }}$ & $0 \mathrm{MVar}$ \\
\hline$\omega_{s}$ & $377 \mathrm{rad} / \mathrm{sec}$ \\
\hline
\end{tabular}

Table 9: Parameters of the grid side inverters

\begin{tabular}{|c|c|}
\hline$K_{p}$ outer loop & 0.4 \\
\hline$K_{i}$ outer loop & 2 \\
\hline$K_{p}$ inner loop & 50 \\
\hline$K_{i}$ inner loop & 100 \\
\hline$L$ & $0.1 \mathrm{H}$ \\
\hline$Q_{\text {ref }}$ & $0 \mathrm{Mvar}$ \\
\hline
\end{tabular}


Rectifier3: $30 \mathrm{~km}$, Inverter1: $60 \mathrm{~km}$, Inverter2: $50 \mathrm{~km}$, Inverter3: $70 \mathrm{~km}$, Cable SR: $100 \mathrm{~km}$.

2. Simplified equivalent DC model for MTDC.

3. Control of wind-side converters.

4. Control of grid-side converters.

5. Voltages at grid side and wind farm side terminals for Case 1.

6. Currents at grid side and wind farm side terminals for Case 1.

7. Droop gain change of grid side terminals for Case 2 .

8. Voltages at grid side and wind farm side terminals for Case 2 .

9. Currents at grid side and wind farm side terminals under optimized operation.

10. Voltages at grid side and wind farm side terminals for fixed droop gains.

11. Voltages at grid side and wind farm side terminals for adaptive droop gains.

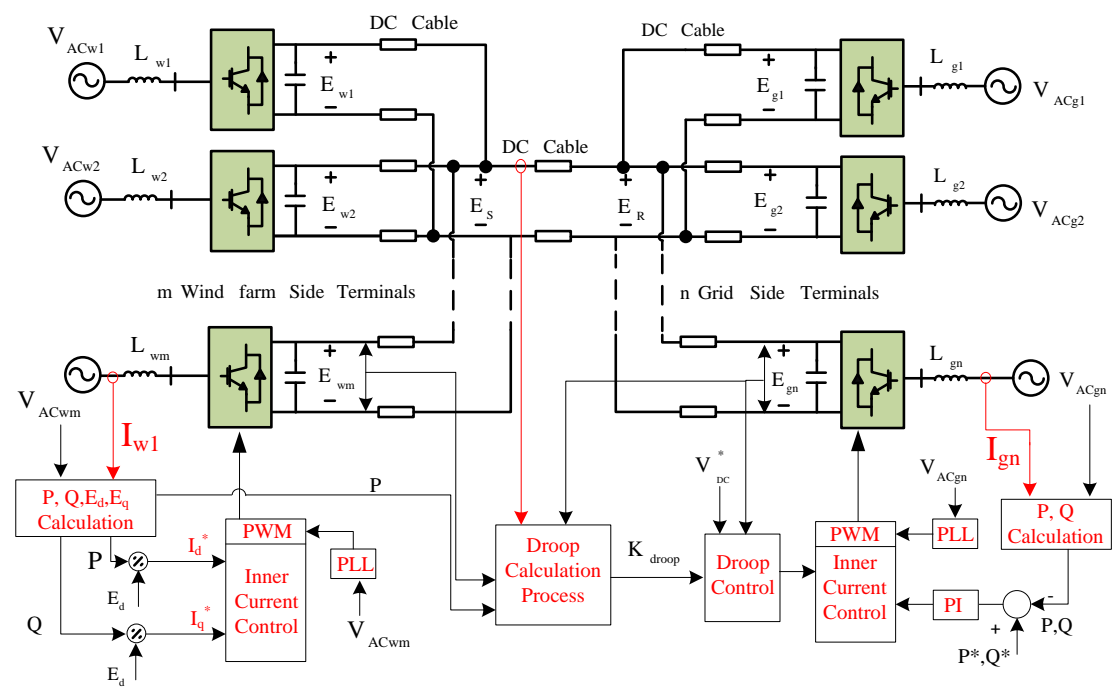

Figure 1: A 6-terminal MTDC. AC voltage level: $100 \mathrm{kV}$; DC voltage level: $250 \mathrm{kV} . C=350 \mu \mathrm{F}$, DC cables have the same resistance: $2.78 \times 10^{-2} \Omega / \mathrm{km}$, DC cable lengths: Rectifier1: 10km, Rectifier2: $20 \mathrm{~km}$, Rectifier3: $30 \mathrm{~km}$, Inverter1: $60 \mathrm{~km}$, Inverter2: $50 \mathrm{~km}$, Inverter3: $70 \mathrm{~km}$, Cable SR: $100 \mathrm{~km}$. 


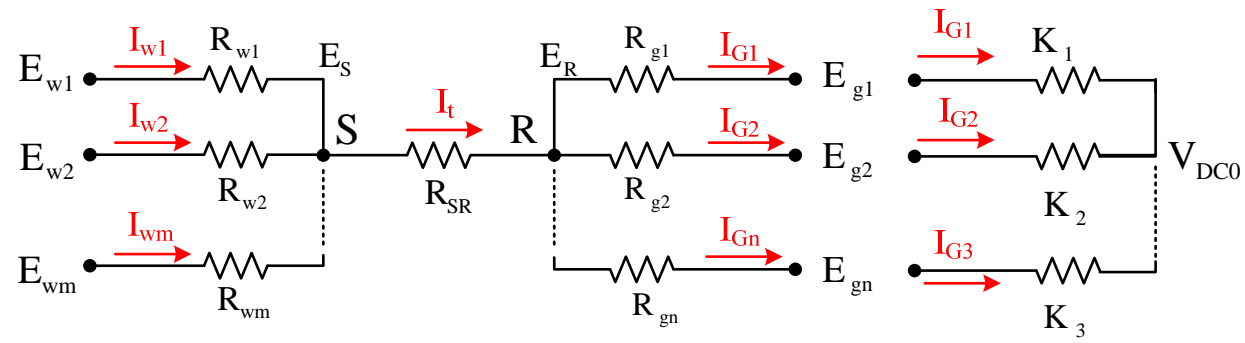

(a)

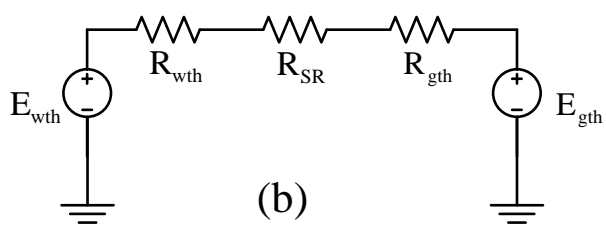

Figure 2: Simplified equivalent DC model for MTDC.

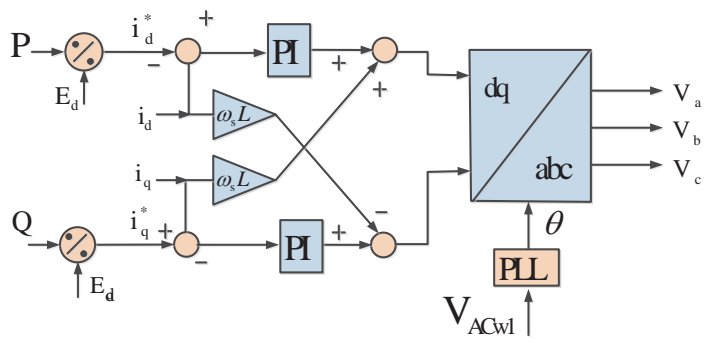

Figure 3: Control of wind-side converters.

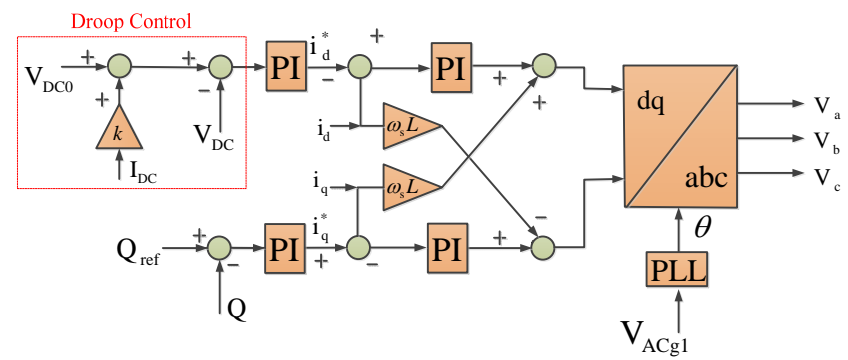

Figure 4: Control of grid-side converters. 

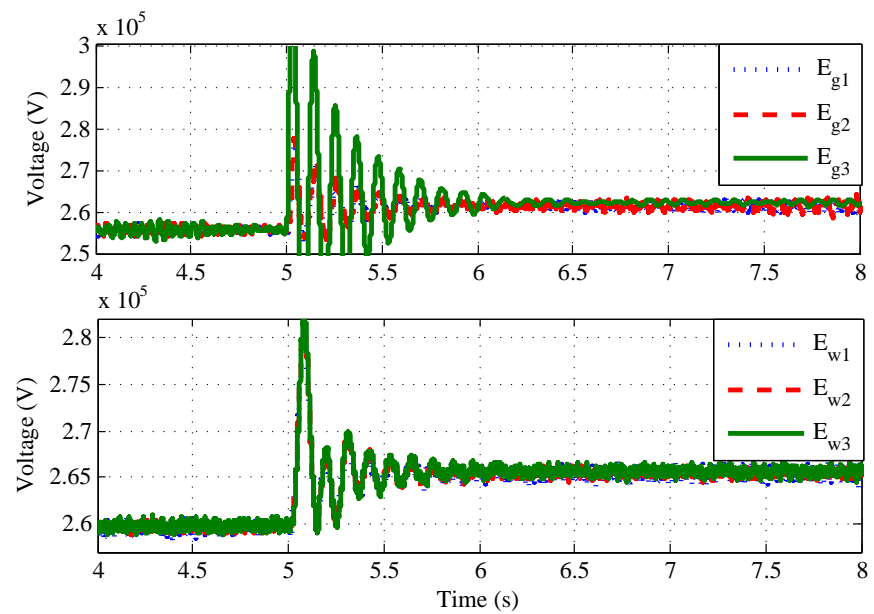

Figure 5: Voltages at grid side and wind farm side terminals for Case 1.

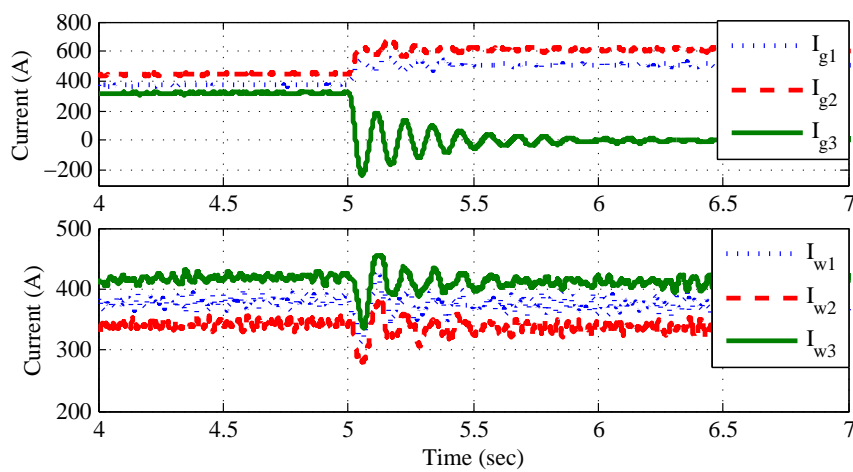

Figure 6: Currents at grid side and wind farm side terminals for Case 1.

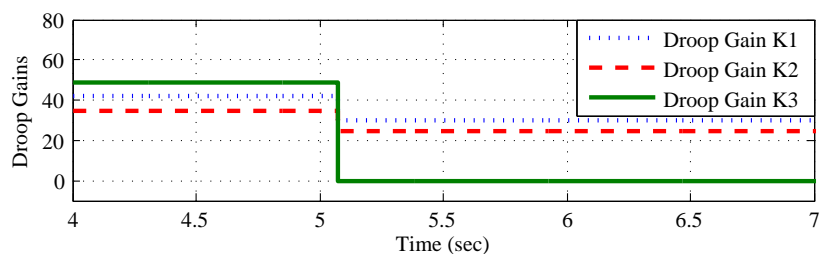

Figure 7: Droop gain change of grid side terminals for Case 2. 

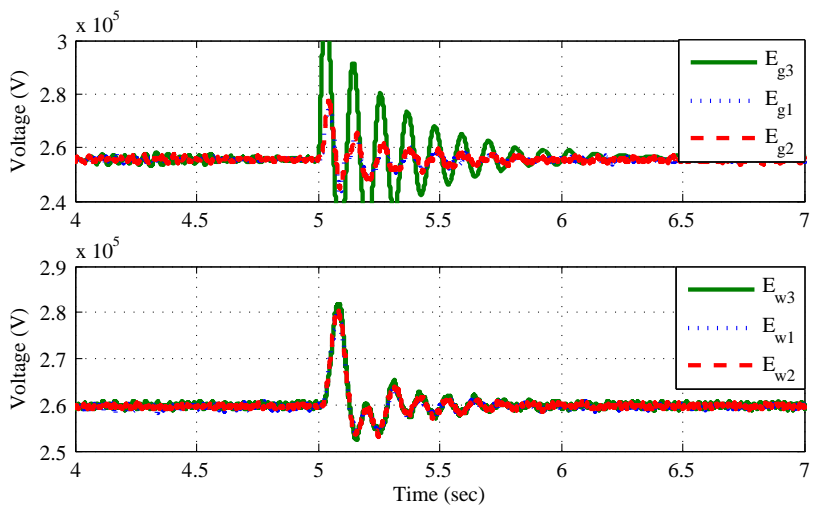

Figure 8: Voltages at grid side and wind farm side terminals for Case 2.
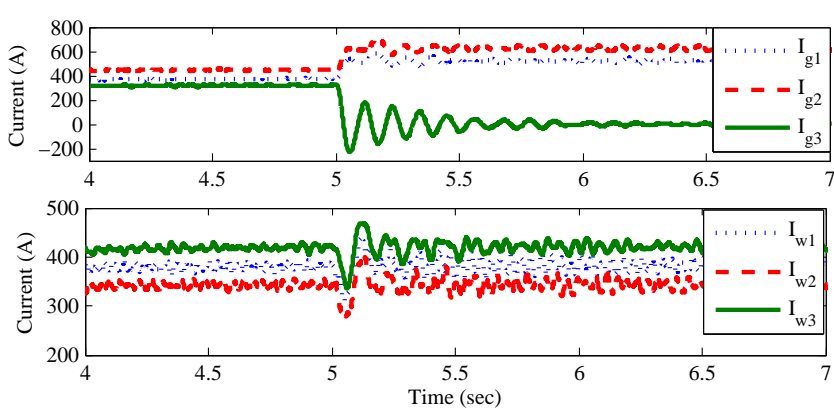

Figure 9: Currents at grid side and wind farm side terminals under optimized operation for case 2.
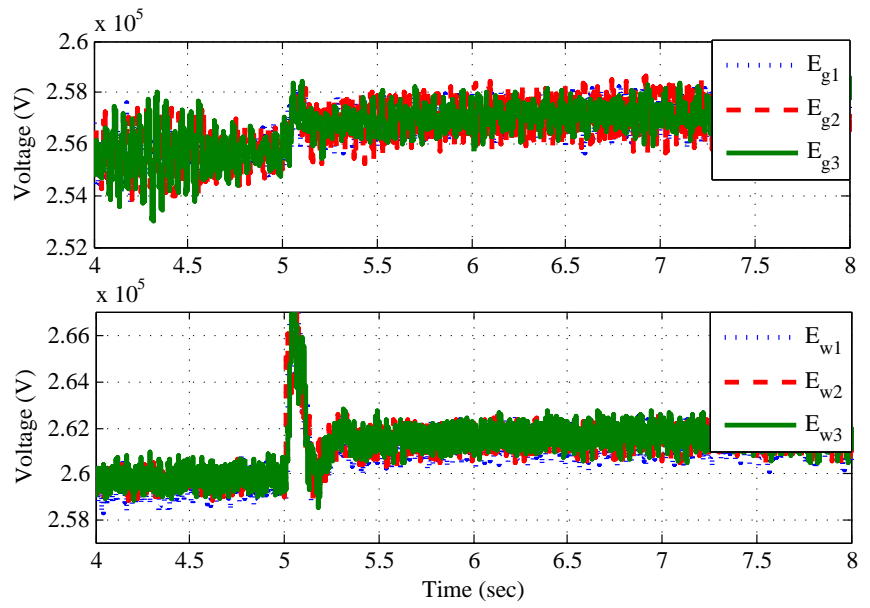

Figure 10: Voltages at grid side and wind farm side terminals for fixed droop gains for case 3. 

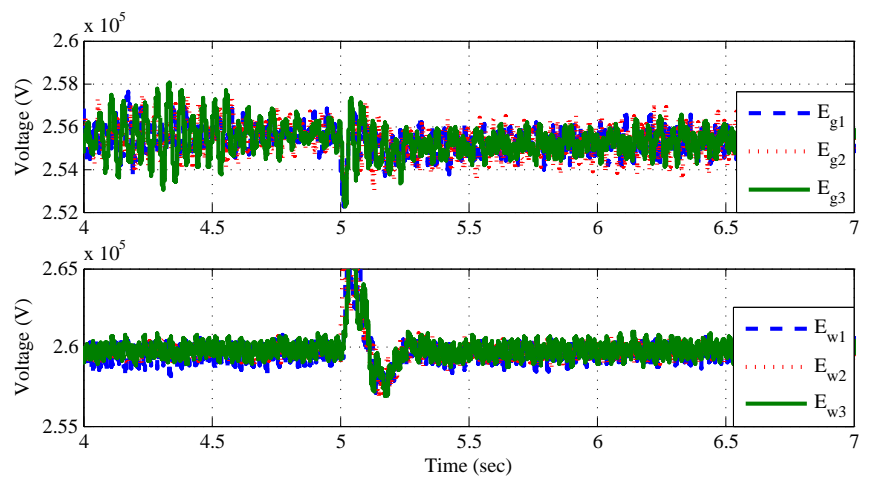

Figure 11: Voltages at grid side and wind farm side terminals for adaptive droop gains for case 4 . 